\title{
Safety and Efficacy of Low-Dosage Apatinib Monotherapy in Advanced Lung Squamous-Cell Carcinoma: A Prospective Cohort Study
}

This article was published in the following Dove Press journal: OncoTargets and Therapy

\author{
Qian Geng ${ }^{1} * *$ \\ Hua Shen ${ }^{2, *}$ \\ Wenyu Zhu' \\ Yingzhi Lu ${ }^{3}$ \\ Mengjie Wang' \\ Hua Jiang' \\ Dongqing Li (iD)
}

'Cancer Center, The Affiliated Changzhou No. 2 People's Hospital of Nanjing Medical University, Changzhou 213000, People's Republic of China; ${ }^{2}$ Sir Run Run Hospital, Nanjing Medical University, Nanjing 210029, People's Republic of China; ${ }^{3}$ Oncology

Department, Lianyungang No. 2 People's Hospital, Lianyungang 222000, People's

Republic of China

*These authors contributed equally to this work
Correspondence: Hua Jiang

Cancer Center, The Affiliated Changzhou No. 2 People's Hospital of Nanjing Medical University, Changzhou 213000, People's Republic of China

Email czeyzlk@hotmail.com

Dongqing Li

Cancer Center, The Affiliated Changzhou

No. 2 People's Hospital of Nanjing

Medical University, Changzhou 213000,

People's Republic of China

Email lidongq1823@I63.com
Background: Lung squamous-cell carcinoma ( $\mathrm{SqCC})$ is the second most common histology in non-small-cell lung carcinomas (NSCLCs). The treatment options for advanced lung SqCC are still an unmet medical need. Apatinib, a small-molecule inhibitor of vascular endothelial growth factor receptor-2 (VEGFR-2), is beneficial in the therapy of advanced NSCLC patients. This study aimed to preliminarily assess the efficacy and safety of lowdosage apatinib in patients with advanced lung SqCC.

Methods: In this single-arm, open-label, investigator-initiated phase II prospective study (ChiCTR1800019808), we enrolled patients aged 54-80 years with platinum-refractory or chemotherapy rejected advanced lung squamous-cell carcinoma. Key exclusion criteria included major blood vessel involvement and gross hemoptysis with an amount of more than $20 \mathrm{~mL}$. Apatinib at an initial dose of $250 \mathrm{mg}$ was administered to patients once daily until disease progression, unacceptable toxicity, withdrawal, or death. The primary endpoint was progression-free survival (PFS) in all patients. We assessed the adverse events according to the treatment received.

Results: Thirty-eight patients were enrolled between June 11, 2015 and August 29, 2018. Two patients failed to evaluate treatment efficacy for personal reasons, and thus 36 patients were eligible for evaluation of tumor response to apatinib. Median PFS was 4.9 months $(95 \%$ CI: 3.0-6.8 months). Six patients achieved partial response (PR); the objective response rate (ORR) was 16.7\% (6/36), and the total disease control rate (DCR) was $77.8 \%(28 / 36)$. Followed up to March 2020, 35 of the 38 patients were dead, and the 1-year survival rate was $21.1 \%$ (8/38). The median overall survival (OS) was 6.9 months (95\% CI: 5.2-8.5 months). The most common adverse events included fatigue (50.0\%), hypertension (42.1\%), proteinuria $(23.7 \%)$, loss of appetite $(23.1 \%)$ and hand-foot reaction $(21.1 \%)$. No grade 4 adverse effect or drug-related mortality occurred.

Conclusions: Low-dose apatinib monotherapy might be an option for patients with advanced lung squamous-cell carcinoma.

Keywords: apatinib, lung squamous-cell carcinoma, VEGF, efficacy, safety

\section{Introduction}

Lung cancer ranks first globally in morbidity and mortality rates among cancers. Lung squamous-cell carcinoma (SqCC), which is included in non-small cell carcinoma (NSCLC), accounts for $25-30 \%$ of lung cancer cases. ${ }^{1}$ Unlike the success obtained in the therapy of adenocarcinoma, the treatment options in advanced lung SqCC are still limited due to the lack of driver mutations and a worse prognosis. ${ }^{2}$ In patients with advanced lung SqCC, platinum-based doublet chemotherapy is the first-line standard 
treatment option. Unfortunately, although the chemotherapy regimen has been updated in the past 30 years, the median survival time is only 1 year for patients with stage IIIB or IV lung SqCC. ${ }^{3}$

Folkman was the first to propose the theory of tumor angiogenesis and the therapeutic conception and mechanism for anti-angiogenesis. ${ }^{4}$ Vascular endothelial growth factor (VEGF) and its receptors, especially VEGF receptor 2 (VEGFR2), are predominantly responsible for angiogenic signaling, ${ }^{5,6}$ which is an essential step in tumor growth, invasion, and metastasis. ${ }^{7}$ Antibodies against VEGF or VEGFR, bevacizumab and ramucirumab were suggested for addition to chemotherapy for advanced non-small-cell lung cancer (NSCLC) without driver mutation. 8,9 Of note, bevacizumab is not approved for the treatment of lung SqCC because of the risk of hemorrhage. ${ }^{10,11}$ To date, scarce evidence exists for the application of monotherapy with antiangiogenesis drugs as second- or further-line therapy for advanced lung SqCC.

As an oral small-molecule tyrosine kinase inhibitor (TKI) available in mainland China, apatinib, which selectively binds to and inhibits VEGFR-2, has a significant effect on advanced gastric and breast cancers. ${ }^{12-14}$ Recent studies showed the efficiency of apatinib in advanced NSCLC patients. ${ }^{15,16}$ In the present pilot investigation, we aimed to explore the efficacy and safety of low-dose apatinib monotherapy in Chinese patients with advanced lung SqCC.

\section{Materials and Methods Study Design and Participants}

We conducted a single-arm, open-label, investigatorinitiated phase II prospective study at the Affiliated Changzhou No.2 People's Hospital of Nanjing Medical University (Changzhou, China) and Lianyungang No.2 People's Hospital (Lianyungang, China). Patients aged 54-80 years, who had platinum-refractory (defined as progression during the initial platinum-based treatment) advanced lung squamous-cell carcinoma (III-IV stage), were eligible to take part in the study. Patients who refused chemotherapy were also eligible.

Key exclusion criteria were major blood vessel involvement and gross hemoptysis with an amount of more than $20 \mathrm{~mL}$. Patients of any one or more of the following conditions were also excluded: severe liver failure, severe kidney failure, and class III or IV cardiac insufficiency.
The study was approved by the Ethics Committee of the Affiliated Changzhou No.2 People's Hospital of Nanjing Medical University, and the patients selected for the study signed consent forms.

\section{Procedures}

Patients were administered apatinib at an initial dose of $250 \mathrm{mg}$ once daily and would not stop until disease progression, unacceptable toxicity, withdrawal, or death.

Dose modifications, including dose interruptions and dose reductions, were allowed for the management of adverse events. Repeated dose interruptions were permitted as required, for a maximum of 14 days on each occasion.

Two dose reductions were permitted for apatinib (250 $\mathrm{mg}$ and $125 \mathrm{mg}$ taken on alternate days, and $125 \mathrm{mg}$ once daily) in the event of toxicity. For grade 4 non-hematological toxicities, apatinib was delayed until recovery to grade 1 or better and then resumed with a reduction of one dose. At the first occurrence of grade 3 non-hematological and grade 3 or 4 hematological toxicities, apatinib was delayed until recovery to grade 1 or below non-hematological and grade 2 or below hematological toxicities, and then treatment was resumed at the same dose. On the second occurrence of grade 3 nonhematological and grade 3 or 4 hematological toxicities, patients underwent one dose reduction (to either $250 \mathrm{mg}$ and $125 \mathrm{mg}$ taken on alternate days or $125 \mathrm{mg}$ once daily, depending on the dose level patients were on when the toxicities occurred). Dose reductions were also recommended for intolerable grade 2 events if investigators considered dose reduction necessary.

Measurable disease was assessed and documented before treatment. Tumor response according to RECIST 1.1 was assessed by investigators using CT scans after 28 days of apatinib monotherapy, and subsequently every 2 months until disease progression was confirmed. Adverse events were evaluated according to the National Cancer Institute Common Toxicity Criteria version 4.0 (CTC 4.0) and were assessed throughout the treatment cycles.

\section{Outcomes}

In all patients, the primary endpoint was progression-free survival (PFS). Secondary endpoints were objective response rate (ORR), disease control rate (DCR), overall survival (OS), and safety. PFS encompassed the time from the first day of apatinib to documented progression or 
death. Objective tumor responses included complete response $(\mathrm{CR})$, partial response $(\mathrm{PR})$, stable disease $(\mathrm{SD})$, and progressive disease (PD). The objective remission rate (ORR) was calculated based on $\mathrm{CR}+\mathrm{PR}$. The disease control rate (DCR) was calculated based on $\mathrm{CR}+\mathrm{PR}+$ SD. OS was defined as the time from the first day of apatinib treatment to death or last follow-up. The followup continued until March 2020.

\section{Statistical Analysis}

We calculated the proportions of patients achieving responses and assessed 95\% CIs using the ClopperPearson method, and the median duration of response and overall survival, and associated 95\% CIs with the Kaplan-Meier method. All statistical analyses were performed using SPSS version 19.0.

\section{Results}

A total of 38 patients were enrolled in the study between June 11, 2015 and August 29, 2018 (Table 1): 97.4\% male, $76.3 \%$ current/former smokers and $50.0 \%$ with ECOG 2-3 performance status. Most patients had received $1(50.0 \%)$ or $\geq 2(39.5 \%)$ prior lines of therapy. Two patients

Table I Baseline Patient Characteristics

\begin{tabular}{|l|l|l|}
\hline & N & Percentage \\
\hline Age & Mean \pm SD & $(67.5 \pm 6.9)$ \\
\hline Sex & & \\
Male & 37 & $97.4 \%$ \\
Female & I & $2.6 \%$ \\
\hline Smoke history & & \\
Yes & 29 & $76.3 \%$ \\
No & 9 & $23.7 \%$ \\
\hline ECOG PS & & \\
0 & 3 & $7.9 \%$ \\
I & 16 & $42.1 \%$ \\
2 & 16 & $42.1 \%$ \\
3 & 3 & $7.9 \%$ \\
\hline Stage & & \\
IIIA & 1 & $2.6 \%$ \\
IIIB & 7 & $18.4 \%$ \\
IIIC & 7 & $18.4 \%$ \\
IV & 23 & $60.5 \%$ \\
\hline Number of prior regimens & & $39.0 \%$ \\
0 & 19 & \\
I & 15 & \\
$\geq 2$ & & \\
\hline
\end{tabular}

Table 2 Treatment Responses

\begin{tabular}{|l|l|l|}
\hline Evaluation & $\mathbf{N}$ & $\%$ \\
\hline CR & 0 & 0 \\
PR & 6 & $16.7 \%$ \\
SD & 22 & $61.1 \%$ \\
PD & 8 & $22.2 \%$ \\
\hline
\end{tabular}

failed to evaluate efficacy for refusing CT examination, so that 36 patients were eligible for evaluation of tumor response to apatinib (Table 2). After apatinib monotherapy, partial response (PR) was achieved in six patients, stable disease (SD) in 22 patients, and progression disease (PD) in eight patients. The objective remission rate (ORR) was $16.7 \%$ (6/36) and the total disease control rate (DCR) was up to $77.8 \%$ (28/36). The median PFS of apatinib was 4.9 months (95\% CI: 3.3-6.5 months) (Figures 1 and 2). The longest PFS was 360 days in a patient ( $250 \mathrm{mg} / \mathrm{d}$ dosage). Followed up to March 2020, 35 of 38 patients were dead, and the one-year survival rate was $21.1 \%(8 / 38)$. The median OS of apatinib was 6.9 months (95\% CI: 5.2-8.5 months) (Figure 3).

All patients were assessed for toxicity. The incidence of adverse events (any grade) regardless of causality was $81.6 \%$ (31/38). No patients discontinued the study. The adverse effects of apatinib treatment included hypertension $(16 / 38$, $42.1 \%)$, proteinuria $(9 / 38,23.7 \%)$, hand-foot reaction $(8 / 38$, $21.1 \%)$, hemoptysis $(7 / 38,18.4 \%)$, fatigue $(19 / 38,50.0 \%)$, neutropenia $(2 / 38,5.3 \%)$, oral mucositis $(4 / 38,10.5 \%)$, heart dysfunction $(1 / 38,2.6 \%)$, abnormal liver function $(1 / 38$, $2.6 \%)$, thrombocytopenia $(1 / 38,2.6 \%)$ and loss of appetite (9/38, 23.7\%; Table 3). Most adverse effects were from Grade 1 to Grade 2, which were tolerable and manageable. No grade 4 adverse effect or drug-related mortality occurred.

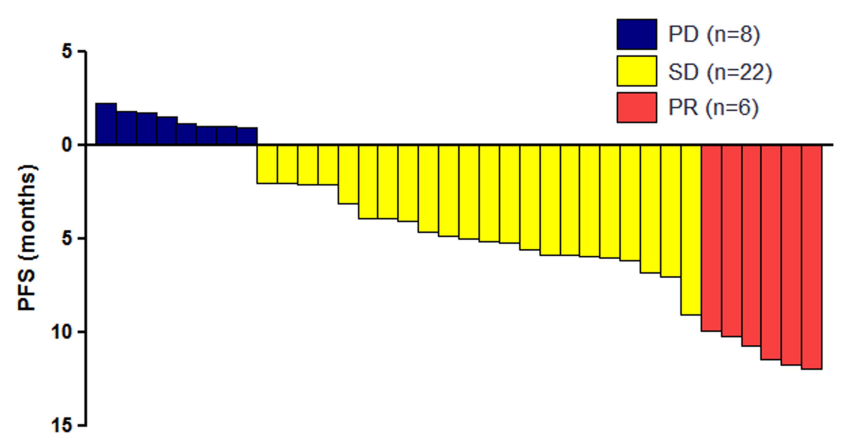

Figure I Waterfall plot for the progression-free survival (PFS) of the 36 patients enrolled in the study. ${ }^{*}$ The columns above the horizontal axis represent the patients who achieved PD (blue), whereas the below represent the patients who achieved PR (red) and SD (yellow). 


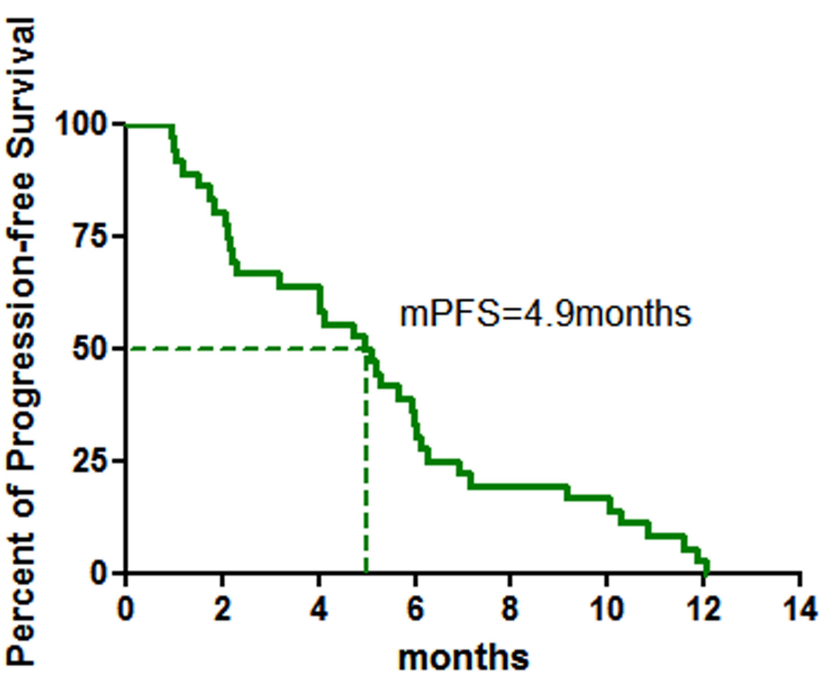

Figure 2 Kaplan-Meier graph for the progression-free survival (PFS) in patients with SqCC $(n=36)$.

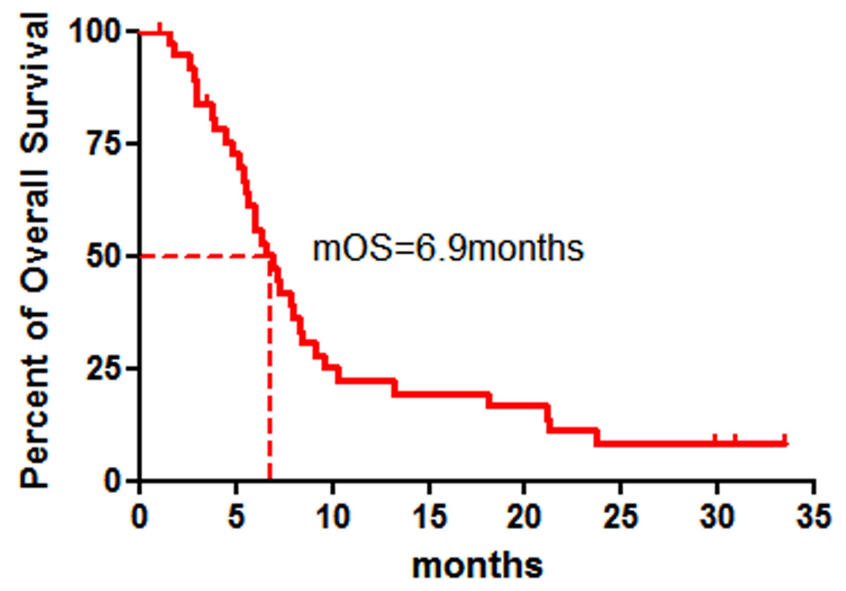

Figure 3 Kaplan-Meier graph for the overall survival (OS) of patients in the study $(n=38)$.

Dose reductions occurred in $5(13.2 \%)$ of 38 patients, of whom $3(60 \%)$ patients required only one dose reduction, and $2(40 \%)$ patients had two dose reductions. Three (60\%) of the five patients had the first dose reduction for apatinib at the completion of the first cycle, and the occurrence of the first dose reduction for apatinib was recorded in two $(40 \%)$ patients during the second cycle.

\section{Discussion}

To our knowledge, up to date, there remains no randomized controlled trial focused on apatinib monotherapy in patients with advanced non-small-cell lung carcinoma. Our study reported, for the first time, the efficacy and safety of apatinib at a low-dosage of 250 or $125 \mathrm{mg}$ daily in patients with platinum-refractory or chemotherapy
Table 3 Possible Treatment-Related Adverse Events

\begin{tabular}{|c|c|c|c|c|}
\hline & $|-| I$ & III & IV & Total \\
\hline \multicolumn{5}{|l|}{ Non-hematological } \\
\hline Hypertension & $10(26.3 \%)$ & $6(15.8 \%)$ & 0 & $16(42.1 \%)$ \\
\hline Proteinuria & 7 (I8.4\%) & $2(5.3 \%)$ & 0 & 9 (23.7\%) \\
\hline Hand-foot reaction & $8(21.1 \%)$ & 0 & 0 & $8(21.1 \%)$ \\
\hline Oral mucositis & $4(10.5 \%)$ & 0 & 0 & $4(10.5 \%)$ \\
\hline Fatigue & 15 (39.5\%) & $4(10.5 \%)$ & 0 & $19(50.0 \%)$ \\
\hline Loss of appetite & 7 (I8.4\%) & $2(5.3 \%)$ & 0 & 9 (23.7\%) \\
\hline Hemoptysis & 7 (I8.4\%) & 0 & 0 & 7 (18.4\%) \\
\hline Heart dysfunction & 0 & I (2.6\%) & 0 & I (2.6\%) \\
\hline Abnormal liver function & I (2.6\%) & 0 & 0 & I (2.6\%) \\
\hline \multicolumn{5}{|l|}{ Hematological } \\
\hline Neutropenia & $2(5.3 \%)$ & 0 & 0 & $2(5.3 \%)$ \\
\hline thrombocytopenia & I (2.6\%) & 0 & 0 & I (2.6\%) \\
\hline
\end{tabular}

rejected advanced lung squamous-cell carcinoma. We found that the ORR and DCR were $16.7 \%$ and $77.8 \%$, and the median PFS and OS were 4.9 and 6.9 months, respectively. Furthermore, most adverse effects were tolerable and manageable.

As an oral anti-angiogenesis drug, apatinib selectively inhibited VEGFR-2 activation. Apatinib was beneficial to increase survival rate as compared with placebo in chemotherapy-refractory advanced gastric cancer patients in a Phase III trial (6.5 vs 4.7 months, $P=0.0149) .{ }^{13}$ Apatinib was also reported to be effective for the treatment of metastatic breast carcinoma patients who failed standard treatment (PFS 3.3 months, OS 10.7 months). ${ }^{14}$ In another study of salvage treatment with apatinib for advanced non-small-cell lung cancer, DCR and ORR were $61.9 \%$ and $9.5 \%$, respectively. ${ }^{15}$ The values of DCR and ORR in the present investigation were $77.8 \%$ and $16.7 \%$, correspondingly, which indicates superior efficacy over docetaxel as second-line chemotherapy in NSCLC. ${ }^{17}$ Of note, half of the patients in the present examination had $P S \geq 2$, which might have an influence on the efficacy of apatinib.

The addition of bevacizumab, a representative for antiangiogenesis drugs, to chemotherapy was beneficial to OS in advanced nonsquamous NSCLC. ${ }^{8}$ However, it has not been approved for the treatment of squamous-cell carcinoma due to severe hemoptysis. ${ }^{10,11}$ Lung SqCC has been a restricted area for anti-angiogenic drugs for a long time. Until 2014, the subgroup analysis in the REVEL study had shown that patients with SqCC seemed to have derived similar benefit from treatment with ramucirumab plus docetaxel without an increase in toxicity, particularly 
respiratory tract hemorrhage, when compared with the placebo group and non-squamous disease group. ${ }^{9}$ The reason that the authors concluded might be that bevacizumab was a monoclonal antibody against VEGF and ramucirumab especially against VEGFR2, which localized to tumor blood vessels.

The combination of ramucirumab and docetaxel had more pronounced beneficial effects than simple chemotherapy in the REVEL study (4.5 vs 3.0 months, $P<0.01$ ). ${ }^{9}$ Few investigations have been focused on the efficiency of anti-angiogenic drugs as monotherapy in advanced lung SqCC. Two clinical cases were reported of NSCLC patients treated with apatinib, with PFS of 4.6 and 6 months. ${ }^{18}$ Another study found that the median PFS for advanced NSCLC patients by using oral apatinib alone as postsecond-line therapy was 4.4 months. In our investigation, the use of apatinib as monotherapy in advanced lung SqCC patients contributed to achieving a median 4.9-month PFS. Previous evidence showed that apatinib exerted its anticancer effect not only via cytotoxicity but also via inhibition of migration and invasion by suppressing the RET/Src signaling pathway. ${ }^{19}$ However, further research on the precise mechanism of action in lung cancer is still required.

Several findings of our study are different from those of previous ones. First, all our patients were with lung $\mathrm{SqCC}$, rather than non-small-cell lung cancer. Thus, our results would be more convincing for the usage of apatinib in advanced lung SqCC treatment. Second, we used apatinib at an initial dose of $250 \mathrm{mg} / \mathrm{d}$, lower than the amount of apatinib applied in previous clinical trials. Zeng et al reported the efficacy of apatinib $(250-425 \mathrm{mg} / \mathrm{d})$ in SCC patients with a median PFS of 3.1 months, ${ }^{20}$ which was significantly less than PFS of our patients. As seen from the results, our patients achieved a median 4.9-month PFS and suffered less adverse effects. Interestingly, in the study of $\mathrm{Hu}$ et al, ${ }^{14}$ where the recommended dose was $500 \mathrm{mg}$ daily, the rate of grade $3 / 4$ toxicity was significantly decreased, but the efficacy was similar to that of the daily regimen of $850 \mathrm{mg}$ in breast carcinoma. Therefore, we suggested that the dosage of apatinib had little effect on its efficacy but was related to the severity of side effects. Further investigation remains to be done to confirm the most suitable dosage of apatinib in patients with lung SqCC. Third, our study reported the median OS simultaneously, which might be a more valuable endpoint than PFS.

The toxicity profile was basically similar to that reported in earlier clinical trials, ${ }^{12-14}$ including hypertension, proteinuria, hand-foot skin reaction, hematological toxicities, fatigue, etc., most of which were grade 1 and 2 . A phase III clinical trial showed that anti-angiogenesis side effects, such as hypertension, proteinuria and handfoot syndrome, often indicated better clinical efficacy among patients with gastric cancer administered with apatinib. ${ }^{13}$ A retrospective cohort study of 269 patients with gastric cancer receiving apatinib in two phase III clinical trials further demonstrated that patients with side effects including hypertension, proteinuria, and hand-foot syndrome had a longer overall survival, progression-free survival, and disease remission rate. ${ }^{21}$ Therefore, these adverse reactions can be used as a basis for the establishment of a prognostic scoring system to predict the efficacy of apatinib. In our study, all six patients achieved PR had one of the three side effects, and none of the patients achieved PD had the above side effects.

However, there are several limitations to our study. First, 38 patients were included in our study, and the efficacy of the treatment could be evaluated in only 36 patients. The results of a larger-scale multicenter study would be more convincing. Second, the patients were treated with apatinib as first to fifth-line therapy based on their different status and willingness. Moreover, herein, we conducted a single-arm, phase II prospective cohort study, not a randomized controlled trail, which would have produced more persuasive results. A randomized placebocontrolled clinical trial needs to be conducted to further validate the efficiency and safety of apatinib in the treatment of lung SqCC patients.

\section{Conclusions}

In conclusion, our study indicated that apatinib might be a therapeutic option in advanced lung squamous-cell carcinoma patients and confirmed that anti-angiogenic therapy is feasible in lung squamous-cell carcinoma. However, further elucidation is needed for the determination of the optimal dosage, patients, and number of treatment lines.

\section{Abbreviations}

Squamous-cell carcinoma (SqCC); non-small cell carcinoma (NSCLC); Vascular endothelial growth factor (VEGF); VEGF receptor 2 (VEGFR2); tyrosine kinase inhibitor (TKI); progression-free survival (PFS); objective response rate (ORR); disease control rate (DCR); overall survival (OS); complete response (CR); partial response $(\mathrm{PR})$; stable disease $(\mathrm{SD})$; progressive disease $(\mathrm{PD})$. 


\section{Data Sharing Statement}

All data generated or analyzed during this study are available from the corresponding author Hua Jiang upon reasonable request.

\section{Ethics Approval and Consent to Participate}

The study was approved by the Ethics Committee of the Affiliated Changzhou No.2 People's Hospital of Nanjing Medical University. All participants provided written informed consent and the procedures were conducted in accordance with the principles of the Declaration of Helsinki.

\section{Consent for Publication}

Not applicable.

\section{Author Contributions}

All authors made a significant contribution to the work reported, whether that is in the conception, study design, execution, acquisition of data, analysis and interpretation, or in all these areas; took part in drafting, revising or critically reviewing the article; gave final approval of the version to be published; have agreed on the journal to which the article has been submitted; and agree to be accountable for all aspects of the work.

\section{Funding}

This study was supported by the Research Fund from Chinese Society of Clinical Oncology-Hengrui (Grant No. Y-HR2015-034), the Research Fund from Jiangsu Health and Family Planning Commission (Grant No. Z201615), and the Research Fund from Changzhou Health and Family Planning Commission (Grant No. ZD201711), the Young Scientists Foundation of Changzhou No.2 People's Hospital (Grant No. 2018K004), the Natural Science Foundation of China (Grant No. 81874230), Jiangsu Social Development Project (Grant No. BE2018726), the Natural Science Foundation of Jiangsu Province (Grants No. BK20171484), the Project of Invigorating Health Care through Science, Technology, and Education (Grant No. Jiangsu Provincial Medical Youth Talent QNRC2016856) and the Summit of the Six Top Talents Program of Jiangsu Province (Grant No. 2017WSN-179).

\section{Disclosure}

The abstract of this paper was presented at the WCLC Conference with the name "Efficacy of low-dosage apatinib monotherapy in treatment of advanced lung squamous cell carcinoma as a poster presentation with interim findings" and was published in "ASCO Abstracts" in JCO Journal with the name "Efficacy and safety of low-dosage apatinib monotherapy in advanced lung squamous cell carcinoma" (DOI: 10.1200/JCO.2018.36.15_suppl.e21170).

The authors declare that they have no conflicts of interest.

\section{References}

1. Dela Cruz C, Tanoue L, Matthay R. Lung cancer: epidemiology, etiology, and prevention. Clin Chest Med. 2011;32:605-644. doi:10.1016/j.ccm.2011.09.001

2. Li T, Kung HJ, Mack PC, Gandara DR. Genotyping and genomic profiling of non-small cell-lung cancer: implications for current and future therapies. J Chin Oncol. 2013;31:1039-1049.

3. Itsudomi T. Advances in target therapy for lung cancer. Jpn J Clin Oncol. 2010;40:101-106. doi:10.1093/jjco/hyp174

4. Folkman J. Tumor angiogenesis: therapeutic implications. $N$ Engl $J$ Med. 1971;285:1182-1186. doi:10.1056/NEJM197111182852108

5. Hicklin DJ, Ellis LM. Role of the vascular endothelial growth factor pathway in tumor growth and angiogenesis. $J$ Clin Oncol. 2005;23:1011-1027. doi:10.1200/JCO.2005.06.081

6. Rosen LS. VEGF-targeted therapy: therapeutic potential and recent advances. Oncologist. 2005;10(6):382-391. doi:10.1634/theoncologist.10-6-382

7. Falcon BL, Chintharlapalli S, Uhlik MT, Pytowski B. Antagonist antibodies to vascular endothelial growth factor receptor 2 (VEGFR-2) as anti-angiogenic agents. Pharmacol Ther. 2016;164:204-225. doi:10.1016/j.pharmthera.2016.06.001

8. Sandler A, Gray R, Perry MC, et al. Paclitaxelcarboplatin alone or with bevacizumab for non-small-cell lung cancer. $N$ Engl $J$ Med. 2006;355:2542-2550.

9. Garon EB, Ciuleanu TE, Arrieta O, et al. Ramucirumab plus docetaxel versus placebo plus docetaxel for second-line treatment of stage IV non-small-cell lung cancer after disease progression on platinum-based therapy (REVEL): a multicentre, double-blind, randomised Phase 3 trial. Lancet. 2014;384:665-673. doi:10.1016/S0140-6736(14)60845-X

10. Johnson DH, Fehrenbacher L, Novotny WF, et al. Randomized phase II trial comparing bevacizumab plus carboplatin and paclitaxel with carboplatin and paclitaxel alone in previously untreated locally advanced or metastatic non-small-cell lung cancer. J Clin Oncol. 2004;22:2184-2191. doi:10.1200/JCO.2004.11.022

11. Sandler AB, Schiller JH, Gray R, et al. Retrospective evaluation of the clinical and radiographic risk factors associated with severe pulmonary hemorrhage in first-line advanced, unresectable non-small-cell lung cancer treated with carboplatin and paclitaxel plus bevacizumab. J Clin Oncol. 2009;27:1405-1412. doi:10.1200/ JCO.2008.16.2412

12. Li J, Qin S, Xu J, et al. Apatinib for chemotherapy-refractory advanced metastatic gastric cancer: results from a randomized, placebo-controlled, parallel-arm, phase II trial. J Clin Oncol. 2013;31:3219-3225. doi:10.1200/JCO.2013.48.8585

13. Li J, Qin S, Xu J, et al. Randomized, double-blind, placebo-controlled phase III trial of apatinib in patients with chemotherapy-refractory advanced or metastatic adenocarcinoma of the stomach or gastroesophageal junction. $J$ Clin Oncol. 2016;34:1448-1454. doi:10.1200/ JCO.2015.63.5995

14. Hu X, Zhang J, Xu B, et al. Multicenter phase II study of apatinib, a novel VEGFR inhibitor in heavily pretreated patients with metastatic triple-negative breast cancer. Int J Cancer. 2014;135:1961-1969. doi:10.1002/ijc.28829 
15. Song ZB, Yu XM, Lou GY, Shi X, Zhang YP. Salvage treatment with apatinib for advanced non-small-cell lung cancer. Onco Targets Ther. 2017;10:1821-1825. doi:10.2147/OTT.S113435

16. Zeng DX, Wang CG, Lei W, Huang JA, Jiang JH. Efficiency of low dosage apatinib in post-first-line treatment of advanced lung adenocarcinoma. Oncotarget. 2017;8(39):66248-66253. doi:10. 18632/oncotarget. 19908

17. Shepherd FA, Dancey J, Ramlau R, et al. Prospective randomized trial of docetaxel versus best supportive care in patients with non-small-cell lung cancer previously treated with platinum-based chemotherapy. J Clin Oncol. 2000;18(10):2095-2103. doi:10.1200/ JCO.2000.18.10.2095

18. Ding L, Li QJ, You KY, Jiang ZM, Yao HR. The use of apatinib in treating nonsmall-cell lung cancer: case report and review of literature. Medicine. 2016;95(20):e3598. doi:10.1097/MD.00000000 00003598
19. Lin C, Wang S, Xie W, Zheng R, Gan Y, Chang J. Apatinib inhibits cellular invasion and migration by fusion kinase KIF5B-RET via suppressing RET/Src signaling pathway. Oncotarget. 2016;7:59236-59244. doi:10.18632/oncotarget.10985

20. Zeng DX, Lei W, Wang CG, Huang JA, Jiang JH. Low dosage of apatinib monotherapy as rescue treatment in advanced lung squamous cell carcinoma. Cancer Chemother Pharmacol. 2019;83:439-442. doi:10.1007/s00280-018-3743-0

21. Liu X, Qin S, Wang Z, et al. Early presence of anti-angiogenesisrelated adverse events as a potential biomarker of antitumor efficacy in metastatic gastric cancer patients treated with apatinib: a cohort study. J Hematol Oncol. 2017;10(1):153. doi:10.1186/s13045-0170521-0

\section{Publish your work in this journal}

OncoTargets and Therapy is an international, peer-reviewed, open access journal focusing on the pathological basis of all cancers, potential targets for therapy and treatment protocols employed to improve the management of cancer patients. The journal also focuses on the impact of management programs and new therapeutic agents and protocols on patient perspectives such as quality of life, adherence and satisfaction. The manuscript management system is completely online and includes a very quick and fair peer-review system, which is all easy to use. Visit http://www.dovepress.com/ testimonials.php to read real quotes from published authors. 